VOL. 75 (2007) [161-168]

\title{
THE HAHN-SCHUR THEOREM ON EFFECT ALGEBRAS
}

\author{
A. Aizpuru, M. Nicasio-Llach and M. Tamayo
}

In this paper we obtain new results on the uniform convergence on matrices and a new version of the matrix theorem of the Hahn-Schur summation theorem in effect algebras.

\section{INTRODUCTION}

In 1936 Birkhoff and Von Neumann ([10]) began to consider the lattice of closed subspaces of a separable infinite-dimensional Hilbert space as a mathematical model for a calculus of quantum logic. From 1980, with Gudder's work ([11]), the quantum mechanical system is given by a probability measure on its set of events that, in general, fails to form a Boolean algebra. To model unsharp quantum logics Foulis and Bennett in 1994 ([7]) introduced effect algebras as follows:

A structure $(L, \oplus, 0,1)$ is called an effect algebra if 0,1 are two distinguished elements and $\oplus$ is a partial binary operation on $L$ which satisfies the following conditions for any $a, b, c \in L$.

(1) $b \oplus a=a \oplus b$ if $a \oplus b$ is defined.

(2) $(a \oplus b) \oplus c=a \oplus(b \oplus c)$ if one side is defined.

(3) For every $a \in L$ there exists a unique $b \in L$ such that $a \oplus b=1$.

(4) If $1 \oplus a$ is defined, then $a=0$.

In [8], the authors study the relation between partially ordered Abelian groups and effect algebras.

Let $(L, \oplus, 0,1)$ be an effect algebra. If $a \oplus b$ is defined we say that $a$ and $b$ are orthogonal. If $a \oplus b=1$ we say that $b$ is the orthocomplement of $a$, and write $b=a^{\prime}$. It is clear that $\mathbf{1}^{\prime}=0,\left(a^{\prime}\right)^{\prime}=a, a \oplus 0$ is defined for all $a \in L$ and $a \oplus 0=a$.

We define now a partial order: $a \leqslant b$ if there exists $c \in L$ such that $a \oplus c=b$. In this case the element $c$ is unique and it will be denoted by $c=b \ominus a$. If $a \leqslant b$ but $a \neq b$ we write $a<b$. We can prove that $a \leqslant b$ if and only if $a \oplus b^{\prime}$ is defined, also $0 \leqslant a \leqslant 1$ if $a \in L$.

The effect algebra $(L, \oplus, 0,1)$ is called a lattice effect algebra if $(L, \leqslant)$ is a lattice. If for $a, b \in L, a \leqslant b$ or $b \leqslant a$ then $(L, \oplus, 0,1)$ is said to be a totally ordered effect algebra;

Received 12th June, 2006

Copyright Clearance Centre, Inc. Serial-fee code: 0004-9727/07 \$A2.00+0.00. 
if for all $a, b \in L, a<b$, there exists $c \in L$ such that $a<c<b$, then $L$ is said to be connected.

Let $F=\left\{a_{i}: 1 \leqslant i \leqslant n\right\}$ be a finite subset of $L$. If $a_{1} \oplus a_{2}$ is defined and $\left(a_{1} \oplus a_{2}\right) \oplus a_{3}, \ldots,\left(a_{1} \oplus a_{2} \oplus \cdots \oplus a_{n-1}\right) \oplus a_{n}$ too, we say that $F$ is orthogonal and we define $\oplus F=\left(a_{1} \oplus a_{2} \oplus \cdots \oplus a_{n-1}\right) \oplus a_{n}$. (by the commutative and associative laws, this sum does not depend on any permutation of elements).

An arbitrary subset of $L, G$ is called orthogonal if $F$ is orthogonal for each $F$, finite subset of $G$. In this case, if the supremum $\bigvee\{\oplus F: F \subset G, F$ is finite $\}$ exists it is called $\oplus$-sum of $G$ and we shall denote it by $\oplus G$.

We say that $L$ is complete if for each orthogonal subset $G$ of $L$, the $\oplus$-sum, $\oplus G$ exists; if for each countable orthogonal subset $G$ of $L$, the $\oplus$-sum exists then we say that $L$ is $\sigma$-complete.

If $(L, \oplus, 0,1)$ is an effect algebra we can consider in $L$ the order topology. Birkhoff, 1948 [9] and Riecanova [13] proved the continuity of $\oplus$ and $\Theta$.

The following is proved in [14]:

(i) If $L$ is totally ordered and $A=\left(a_{i}\right)_{i}$ is orthogonal $\oplus$-summable, then $\left\{a_{n}\right\}_{n \in \mathbb{N}}$ is order convergent to 0 .

(ii) If $L$ is $\sigma$-complete, $\left(a_{i}\right)_{i}$ and $\left(b_{i}\right)_{i}$ are two orthogonal $\oplus$-summable sequences of $L$ and for each $i \in \mathbb{N}, b_{i} \leqslant a_{i}$, then we have:

$$
\bigvee_{n \in \mathbb{N}}\left\{\bigoplus_{i=1}^{n}\left(a_{i} \ominus b_{i}\right)\right\}=\bigvee_{n \in \mathbb{N}}\left\{\bigoplus_{i=1}^{n} a_{i}\right\} \ominus \bigvee_{n \in \mathbb{N}}\left\{\bigoplus_{i=1}^{n} b_{i}\right\}
$$

(iii) If $L$ is $\sigma$-complete, totally ordered and connected, then for each $h \in L$, $0<h$, there exists an orthogonal $\oplus$-summable sequence $\left(h_{i}\right)_{i}$ of $L$ such that

$$
\bigvee_{n}\left\{\bigoplus_{i=1}^{n} h_{i}\right\}<h
$$

Some interesting results about matrix convergence theorem in quantum logics are studied in $[14,16,15,12]$. These results generalise the results in [6]. In this paper, we obtain a new version of the Hahn-Schur summation theorem in effect algebras. This version can have direct applications to measure theory on effect algebras $([3,4])$. Some similar results for normed spaces are obtained in $[1]$.

\section{MAIN THEOREM AND ITS PROOF}

Let $(L, \oplus, 0,1)$ be a totally ordered effect algebra.

We say that ([14]) the sequence $\left(a_{n}\right)_{n}$ of $L$ is a Cauchy sequence if for each $h \in L$, $0<h$, there exists $n_{0} \in \mathbb{N}$ such that when $n_{0} \leqslant n, n_{0} \leqslant m$, if $a_{n} \leqslant a_{m}$ then $a_{m} \ominus a_{n}<h$ and if $a_{m} \leqslant a_{n}$, then $a_{n} \ominus a_{m}<h$. 
In the next remark we obtain results that will be useful later.

If $L$ is an effect algebra, then $L$ is a $D$-algebra ([13]) and the following is satisfied: if $b \ominus a$ and $c \ominus b$ are defined then also $c \ominus a$ and $(c \ominus a) \Theta(c \ominus b)$ are defined and $(c \ominus a) \ominus(c \ominus b)=b \ominus a$.

Let $(L, \oplus, 0,1)$ be a totally ordered effect algebra.

If $a, b \in L$, by $a-b$ we mean $a \ominus b$ if $b \leqslant a$ and $b \ominus a$ if $a \leqslant b$.

If $\{a, b, c\} \subset L$ then $c-a \leqslant(b-a) \oplus(c-b)([5])$.

DEFinition 0.1: Let $(L, \oplus, 0,1)$ be a totally ordered effect algebra. An orthogonal sequence $\left(a_{i}\right)_{i}$ is called an unconditionally Cauchy series if for each $h>0$ there exists $n_{0}$ such that when $n \geqslant n_{0}$ and $B \subset\{n, n+1, \ldots\}$ is finite then $\bigoplus_{i \in B} a_{i}<h$.

We shall denote by $\phi_{0}(\mathbb{N})$ the set of finite subsets of $\mathbb{N}$. Every subset $\mathcal{F}$ of $\mathcal{P}(\mathbb{N})$ such that $\phi_{0}(\mathbb{N}) \subset \mathcal{F}$ will be called a natural family.

A natural family $\mathcal{F}$ is subsequentially complete $(S C)$ if for every sequence $\left(A_{i}\right)$ of disjoints sets of $\mathcal{F}$ there exists $M \subset \mathbb{N}$ infinite such that $\bigcup_{i \in M} A_{i} \in \mathcal{F}$.

The following result is a new version of the Hahn-Schur theorem and it is proved in [14]:

Let $(L, \oplus, 0,1)$ be a $\sigma$-complete totally ordered connected effect algebra, $a_{i j} \in L$ for $i, j \in \mathbb{N}$ such that $\left(a_{i j}\right)_{j}$ is an orthogonal sequence of $L$ for each $i \in \mathbb{N}$. If for each subset $A$ of $\mathbb{N}$, the $\oplus$-sum sequence $\left(\bigoplus_{j \in A} a_{i j}\right)_{i}$ is convergent, then $\left(a_{i j}\right)_{j \in \mathbb{N}}$ are uniformly $\oplus$-summable with respect to $i \in \mathbb{N}$.

We shall generalise this result using natural families, which can be non- $S C$.

In [1] the following definition is introduced:

DEFINITION 0.2: Let $\mathcal{F}$ be a natural family, we shall say that $\mathcal{F}$ is $S$ if for every pair $\left[\left(A_{i}\right)_{i},\left(B_{i}\right)_{i}\right]$ of disjoint sequences of mutually disjoint sets of $\phi_{0}(\mathbb{N})$ there exists $M \subset \mathbb{N}$ infinite and $B \in \mathcal{F}$ such that if $i \in M$ then $A_{i} \subset B$ and $B \cap B_{i}=\varnothing$.

REMARK. In [2] it is proved that there exist natural families being $S$ but not $S C$.

REMARK. Let $L$ be a totally ordered effect algebra and let $\left\{a_{1}, \ldots, a_{n}\right\},\left\{b_{1}, \ldots, b_{n}\right\}$ be two orthogonal sets of $n$ elements of $L$. In [14] it is proved that

$$
\left(\bigoplus_{i=1}^{n} a_{i}\right)-\left(\bigoplus_{i=1}^{n} b_{i}\right)=\left(\bigoplus_{i \in A_{+}}\left(a_{i} \ominus b_{i}\right)\right) \ominus\left(\bigoplus_{i \in A_{-}}\left(b_{i} \ominus a_{i}\right)\right)
$$

where $A_{+}=\left\{i \leqslant n: b_{i} \leqslant a_{i}\right\}, A_{-}=\{1, \ldots, n\} \backslash A_{+}$.

If $L$ is $\sigma$-complete and $\left(a_{i}\right)_{i},\left(b_{i}\right)_{i}$ are orthogonal sequences we have that:

$$
\left(\bigoplus_{i=1}^{\infty} a_{i}\right)-\left(\bigoplus_{i=1}^{\infty} b_{i}\right)=\left(\bigoplus_{i \in A_{+}}\left(a_{i} \ominus b_{i}\right)\right) \ominus\left(\bigoplus_{i \in A_{-}}\left(b_{i} \ominus a_{i}\right)\right)
$$


where $A_{+}=\left\{i \in \mathbb{N}: b_{i} \leqslant a_{i}\right\}, A_{-}=\left\{i \in \mathbb{N}: a_{i}<b_{i}\right\}$

If $(L, \oplus, 0,1)$ is a totally ordered effect algebra and $\left(a_{i}\right)_{i}$ is an unconditional Cauchy series then $\left(a_{i}\right)_{i}$ converges to zero. If $\bigoplus_{i \in \mathbb{N}} a_{i}$ exists then it is easy to prove that $\left(a_{i}\right)_{i}$ is an unconditional Cauchy series.

The next theorem is similar to a result of [1] valid in normed spaces:

THEOREM 1. (Hahn-Schur Summation) Let $(L, \oplus, 0,1)$ be a $\sigma$-complete totally ordered connected effect algebra. Let $\left(a_{i j}\right)$ be a matrix in $L$ such that the rows are orthogonal sequences and the columns are Cauchy sequences. The following assertions are equivalent:

1. There exists a natural family $\mathcal{F}$ with the property $S$ such that $\left(\bigoplus_{j \in B} a_{i j}\right)_{i}$ has the Cauchy property if $B \in \mathcal{F}$.

2. If $\left(A_{n}\right)_{n}$ is a sequence of disjoint subsets of $\phi_{0}(\mathbb{N})$ then $\left(\bigoplus_{j \in A_{n}} a_{i j}\right)_{i}$ has the Cauchy property uniformly in $n \in \mathbb{N}$.

3. $\bigoplus_{j \in \mathbb{N}} a_{i j}$ is uniformly and unconditional Cauchy series in $i \in \mathbb{N}$.

4. $\bigoplus_{j \in A} a_{i j}$ is uniformly an unconditional Cauchy series in $i \in \mathbb{N}$ and $A \in \mathcal{P}(\mathbb{N})$

5. $\left(\bigoplus_{j \in A} a_{i j}\right)_{i}$ has the Cauchy property if $A \in \mathcal{P}(\mathbb{N})$.

Proof: We suppose that 1 is true and 2 is false. Then, there exists $h>0$ such that for each $k$ there exist $i>k$ and $n_{k}$ such that $\underset{j \in A_{n_{k}}}{\bigoplus} a_{k j}-\underset{j \in A_{n_{k}}}{\bigoplus} a_{i j}>h$. Also, it is easy to show that for each $k \in \mathbb{N}$ and each $m \in \mathbb{N}$ there exist $i>k$ and $n_{k}$ such that $\bigoplus_{j \in A_{n_{k}}} a_{k j}-\bigoplus_{j \in A_{n_{k}}} a_{i j}>h$ and $A_{n_{k}} \cap\{1, \ldots, m\}=\emptyset$.

Let $\left\{h_{1}, h_{2}, h_{3}, h_{4}\right\} \subset L \backslash\{0\}$ be such that $h_{1} \oplus h_{2} \oplus h_{3} \oplus h_{4}<h$.

For $k_{1}=1$, there exist $i_{1}$ and $k_{1}$ and $n_{1}$ such that $\underset{j \in A_{n_{1}}}{\bigoplus} a_{k_{1} j}-\bigoplus_{j \in A_{n_{2}}} a_{i_{2} j}>h$.

Since $\bigoplus_{j} a_{k_{2} j}$ and $\bigoplus_{j} a_{i_{1} j}$ are an unconditional Cauchy series, we have that there exists $m_{1}$ such that $\bigoplus_{j \in B} a_{k_{1} j}<h_{1}$ and $\bigoplus_{j \in B} a_{i_{1} j}<h_{2}$ if $B \subset\left\{m_{1}+1, \ldots\right\}$ is finite.

We observe that if $B \subset\left\{m_{1}+1, \ldots\right\}$ and $B^{+} \subset\left\{j \in B: a_{i_{1} j} \leqslant a_{k_{1} j}\right\}$ and $B^{-}$ $\subset\left\{j \in B: a_{k_{1} j} \leqslant a_{i_{1} j}\right\}$ then

$$
\bigoplus_{j \in B} a_{k_{1} j}-\bigoplus_{j \in B} a_{i_{1} j}=\left[\bigoplus_{j \in B^{+}}\left(a_{k_{1} j}-a_{i_{1} j}\right)\right]-\left[\bigoplus_{j \in B^{-}} a_{i_{1} j}-a_{k_{1} j}\right]<h_{1}+h_{2}
$$

because

$$
\begin{aligned}
& \bigoplus_{j \in B^{+}}\left(a_{k_{1} j}-a_{i_{1} j}\right) \leqslant \bigoplus_{j \in B^{+}} a_{k_{1} j}<h_{1} \\
& \bigoplus_{j \in B^{-}}\left(a_{i_{1} j}-a_{k_{1} j}\right) \leqslant \bigoplus_{j \in B^{-}} a_{i_{1} j}<h_{2}
\end{aligned}
$$


As for $j \in\left\{1, \ldots, m_{1}\right\},\left(a_{i j}\right)_{i}$ has the Cauchy property, we can consider $\left\{h_{31}, \ldots, h_{3 m_{1}}\right\}$ $\subset L \backslash\{0\}$ such that if $j \in\left\{1, \ldots, m_{1}\right\}$ and $p, q \geqslant i_{0}$ is $x_{p j}-x_{q j}<h_{1 j}$. So if $C \subset\left\{1, \ldots, m_{1}\right\}$ and $C^{+}=\left\{j \in C: a_{p j}<a_{q j}\right\}$ we have that $\bigoplus_{j \in c^{+}}\left(a_{p j}-a_{q j}\right)<h_{3}, \underset{j \in C^{-}}{\bigoplus}\left(a_{q j}-a_{p j}\right)<h_{3}$ and it will be also $\left(\bigoplus_{j \in C} a_{p j}\right)-\left(\bigoplus_{j \in C} a_{q j}\right)<h_{3}$.

Take $k_{2}>i_{01}$ and $i_{2}>k_{2}$ and $n_{2}$ such that $\left(\bigoplus_{j \in A_{n_{2}}} a_{k_{2} j}\right)-\left(\bigoplus_{j \in A_{n_{2}}} a_{i_{2} j}\right)>h$ and $A_{n_{2}} \cap\left\{1, \ldots, m_{1}\right\}=\emptyset$.

We obtain, inductively, the sequences of integers $k_{1}<i_{1}<k_{2}<i_{2}<\cdots<k_{r}$ $<i_{r}<\cdots$ and $m_{1}<m_{2}<\cdots<m_{r}<\cdots$ such that, if $r \in \mathbb{N}$ we have:

i.- $\bigoplus_{j \in C^{+}}\left(a_{k_{r} j}-a_{i_{r} j}\right)<h_{3}, \bigoplus_{j \in C^{-}}\left(a_{i_{r} j}-a_{k_{r} j}\right)<h_{3}$ if $C \subset\left\{1, \ldots, m_{r-1}\right\}$ and $C^{+}=\left\{j \in C: a_{i_{r} j} \leqslant a_{k_{r} j}\right\}, C^{-}=\left\{j \in C: a_{k_{r} j}<a_{i_{r} j}\right\}$.

ii.- $\quad \bigoplus_{j \in A_{n_{r}}} a_{k_{r} j}-\bigoplus_{j \in A_{n_{r}}} a_{i_{r} j}>h$ and $m_{r-1}<\inf A_{n_{r}} \leqslant \sup A_{n_{r}}<m_{\tau}$.

iii.- If $B \subset\left\{m_{r+1}, \ldots\right\}$ is finite and $B^{+}=\left\{j \in B: a_{i_{r} j} \leqslant a_{k_{r}}\right\}$ and $B^{-}=\{j$ $\left.\in B: a_{k_{r} j}<a_{i_{r} j}\right\}$ then $\bigoplus_{j \in B^{+}}\left(a_{k_{r} j}-a_{i_{r} j}\right)<h_{1}, \bigoplus_{j \in B^{-}}\left(a_{i_{r} j}-a_{k_{r} j}\right)<h_{2}$ and $\left(\bigoplus_{j \in B} a_{k_{r} j}\right)-\left(\bigoplus_{j \in B} a_{i_{r} j}\right)<h_{1}+h_{2}$

For each $r \in \mathbb{N}$, let $B_{n_{r}}=\left(m_{r-1}, m_{r} \backslash \backslash A_{n_{r}}\right.$, we have a pair of disjoint sequences of disjoint sets of $\phi_{0}(\mathbb{N}):\left[\left(A_{n_{r}}\right),\left(B_{n_{r}}\right)\right]_{r}$; since the family $\mathcal{F}$ has the $S$ property, there exists $M \subset \mathbb{N}$ infinite and $A \in \mathcal{F}$ such that if $r \in M$ then $A_{n_{r}} \subset A$ and $A \cap B_{n_{r}}=\emptyset$. We have that $\left(\bigoplus_{j \in B} a_{i j}\right)_{i}$ has the Cauchy property. Then there exists $i_{0}$ such that

$$
\left(\bigoplus_{j \in A} a_{p j}\right)-\left(\bigoplus_{j \in A} a_{q j}\right)<h_{4}
$$

if $p, q \geqslant i_{0}$.

Let $r$ be such that $i_{0}<k_{r}$, we have that $a=\left(\bigoplus_{j \in A} a_{k_{r} j}\right)-\left(\bigoplus_{j \in A} a_{i_{r} j}\right)$.

Let $A^{+}=\left\{j \in A: a_{i_{r} j} \leqslant a_{k_{r} j}\right\}$ and $A^{-}=\left\{j \in A: a_{k_{r} j}<a_{i_{r} j}\right\}$. We have that

$$
\begin{aligned}
a^{+} & =\bigoplus_{j \in A^{+}}\left(a_{k_{r} j}-a_{i_{r} j}\right) \\
& =\left[\bigoplus_{\substack{j \in A^{+} \\
j \leqslant m_{r-1}}}\left(a_{k_{r} j}-a_{i_{r} j}\right)\right] \oplus\left[\bigoplus_{j \in A_{n_{r}} \cap A^{+}}\left(a_{k_{r} j}-a_{i_{r} j}\right)\right] \oplus\left[\bigoplus_{\substack{j \in A^{+} \\
j>m_{r}}}\left(a_{k_{r} j}-a_{i_{r} j}\right)\right]=b^{+} \oplus c^{+}
\end{aligned}
$$

where $c^{+}=\bigoplus_{j \in A_{n_{r}} \cap A^{+}}\left(a_{k_{r} j}-a_{i_{r} j}\right)$ and

$$
b^{+}=\left[\bigoplus_{\substack{j \in A^{+} \\ j \leqslant m_{r-1}}}\left(a_{k_{r} j}-a_{i_{r} j}\right)\right] \oplus\left[\bigoplus_{\substack{j \in A^{+} \\ j>m_{r}}}\left(a_{k_{r} j}-a_{i_{r} j}\right)\right]<h_{1}+h_{3} .
$$


Analogously, $a^{-}=b^{-} \oplus c^{-}$, where $a^{-}=\bigoplus_{j \in A^{-}}\left(a_{i_{r} j}-a_{k_{r} j}\right)$ and $c^{-}=\bigoplus_{j \in A^{-} \cap A_{n_{r}}}\left(a_{i_{r} j}-a_{k_{r} j}\right)$ and

$$
b^{-}=\left[\bigoplus_{\substack{j \in A^{-} \\ j \leqslant m_{r-1}}}\left(a_{i_{r} j}-a_{k_{r} j}\right)\right] \oplus\left[\bigoplus_{\substack{j \in A^{-} \\ j>m_{r}}}\left(a_{i_{r} j}-a_{k_{r} j}\right)\right] .
$$

We also have that $b^{-}<h_{2}+h_{3}$.

If we consider $c^{+}, a^{+}, c^{-}$we deduce that $c^{+} \ominus c^{-}$is less or equal than $b^{+} \oplus\left(a^{+}-c^{-}\right)$. If we consider $a^{+}, a^{-}, c^{-}$we deduce that $a^{+} \ominus c^{-}$is less or equal than $b^{-} \oplus\left(a^{+}-a^{-}\right)$.

So we deduce that $\bigoplus_{j \in A_{n_{r}}} a_{k_{r} j}-\bigoplus_{j \in A_{n_{r}}} a_{i_{r} j}=c^{+}-c^{-}$is less or equal than $h$, that is a contradiction.

Now we are going to prove that $2 \Rightarrow 3$. If 2 is true and 3 is false, we have that there exists $h>0$ such that for each $n$ there exists $B \subset\{n+1, \ldots\}$ finite and $i_{n} \in \mathbb{N}$ such that $\bigoplus_{j \in B} a_{i_{n} j}>h$.

We consider $h_{1} \in L \backslash\{0\}$ such that $h_{1}<h$. For $n_{1}=1$ there exists $i_{1}$ and $B_{1}$ $\subset\{1,2, \ldots\}$ finite such that $\bigoplus_{j \in B_{1}} a_{i j}>h$. For $i_{1}$ there exists $m_{1}>\sup B_{1}$ such that $\bigoplus_{j \in B} a_{i j}$ $<h_{1}$ if $B \subset\left\{m_{1}+1, \ldots\right\}$. For $m_{1}$ there exists $i_{2}$ and $B_{2} \subset\left\{m_{1}+1, \ldots\right\}$ finite such that $\bigoplus_{j \in B_{2}} a_{i_{2} j}>h$.

Inductively we determine two sequences of integers $i_{1}<i_{2}<\cdots<i_{r}<\cdots, m_{1}$ $<m_{2}<\cdots<m_{r}<\cdots$ and a sequence $\left(B_{r}\right)_{r}$ of pairwise disjoint sets of $\phi_{0}(\mathbb{N})$ such that, if $r>1$ :

$$
\text { i.- } \quad B_{r} \subset\left\{m_{r-1}+1, \ldots\right\} \text { and } \bigoplus_{j \in B_{r}} a_{i_{r} j}>h \text {. }
$$

ii.- $\bigoplus_{j \in B} a_{i_{q} j}<h$ if $B \subset\left\{m_{r-1}+1, \ldots\right\}$, so for each $r>1$ we have $\bigoplus_{j \in B_{r-1}} a_{i_{r+1} j}$ - $\bigoplus_{j \in B_{r+1}} a_{i_{r} j}>h-h_{1}$ and this contradicts that $\left(\bigoplus_{j \in B_{r}} a_{i j}\right)_{i}$ has Cauchy property uniformly in $r \in \mathbb{N}$.

$3 \Rightarrow 4$ and $5 \Rightarrow 1$ are trivial. We are going to prove $4 \Rightarrow 5$.

We suppose that there exists $A \in \mathcal{P}(\mathbb{N})$ such that $\left(\bigoplus_{j \in A} a_{i j}\right)_{i}$ is not a Cauchy sequence. We have that there exists $h>0$ such that for each $n$ there exist $i, k$ such that $n<i<k$ and $\bigoplus_{j \in A} a_{k j}-\bigoplus_{j \in A} a_{i j}>h$.

For $n=1$ there exist: $i_{1}, k_{1}$ such that $1<i_{1}<k_{1}$ and $\bigoplus_{j \in A} a_{k j}-\bigoplus_{j \in A} a_{i j}>h$.

Inductively, we obtain sequences $k_{1}<i_{1}<k_{2}<i_{2}<\cdots<k_{r}$ $<i_{r}<\cdots$ such that $\bigoplus_{j \in A} a_{k_{r} j}-\bigoplus_{j \in A} a_{i_{r}}$, if $r \in \mathbb{N}$.

Let $h_{1}, h_{2} \in L \backslash\{0\}$ be such that $h_{1}+h_{2}<h_{3}$.

By hypothesis, there exists $m \in \mathbb{N}$ such that if $B \subset\{m+1, \ldots\} \cap A$ then $\bigoplus_{j \in B} a_{i j}<h_{1}$ for each $i \in \mathbb{N}$. 
We have that, for $j \in\{1, \ldots, m\} \cap A,\left(a_{i j}\right)_{i}$ is a Cauchy sequence and it will exist $i_{0}$ such that, if $p, q \geqslant i_{0}$ then $a_{p j}-a_{q j} \leqslant h_{2 j}$, where $\left\{h_{21}, \ldots, h_{2 m}\right\} \subset L \backslash\{0\}$ and $h_{21} \oplus \cdots \oplus h_{2 m}<h_{2}$. So, if $c \subset\{1, \ldots, m\} \cap A$ and $p, q \geqslant i_{0}$, then $\bigoplus_{j \in c^{+}}\left(a_{p j}-a_{q j}\right)<h_{1}, \bigoplus_{j \in c^{-}}\left(a_{q j}-a_{p j}\right)<h_{1}$ and $\bigoplus_{j \in c}\left(a_{p j}-a_{q j}\right)<h_{1}$, where $c^{+}=\left\{j \in c: a_{q j} \leqslant a_{p j}\right\}$ and $c^{-}=\left\{j \in c: a_{p j} \leqslant a_{q j}\right\}$.

Let $r$ be such that $i_{0}<k_{r}<i_{r}$. If $a^{+}=\bigoplus_{j \in A^{+}}\left(a_{k_{r} j}-a_{i_{r} j}\right)$ and $a^{-}=\bigoplus_{j \in A^{-}}\left(a_{i_{r} j}-a_{k_{r} j}\right)$, where $A^{+}=\left\{j \in A: a_{i_{r} j} \leqslant a_{k_{r}}\right\}$ and $A^{-}=\left\{j \in A: a_{k_{r} j} \leqslant a_{i_{r}}\right\}$, we deduce

$$
a^{+}=\left[\bigoplus_{\substack{j \in A^{+} \\ j \leqslant m}}\left(a_{k_{r} j}-a_{i_{r} j}\right)\right] \oplus\left[\bigoplus_{\substack{j \in A^{+} \\ j>m}} a_{k_{r} j}-a_{i_{r} j}\right]<h_{1}+h_{2} .
$$

Analogously, we have that $a^{-}<h_{1}+h_{2}$. So $\bigoplus_{j \in A}\left(a_{k_{r} j}-a_{i_{r} j}\right)=a^{+} \ominus a^{-}<h$ and this is a contradiction.

REMARK. In the same conditions of the last theorem, we shall have that the rows $\left(a_{i j}\right)_{j}$ have uniformly the Cauchy property and we can deduce from [5] that the columns also have uniformly the Cauchy property, from this it is easy to deduce that given $h \in L \backslash\{0\}$ there exist $i_{0}, j_{0}$ such that $a_{i j}<h$ if $i \geqslant i_{0}$ and $j \geqslant j_{0}$. In other words, $\lim a_{i j}=0$ in the sense of Pringsheim.

\section{REFERENCES}

[1] A. Aizpuru and A. Gutiérrez-Dávila, 'Unconditionally Cauchy series and uniform convergence on Matrices', Chinese Ann. Math. Ser. B 25 (2004), 335-346.

[2] A. Aizpuru and A. Gutiérrez-Dávila, 'On the interchange of series and applications', Bull. Belg. Math. Soc. 11 (2004), 409-430.

[3] A. Aizpuru, A. Gutiérrez-Dávila and J. Wu, 'Measures defined on quantum logics of sets', Internat. J. Theoret. Phys. 44 (2005), 1451-1458.

(4) A. Aizpuru and M. Tamayo, 'Classical properties of measure theory on effect algebras', Fuzzy Sets and Systems 157 (2006), 2139-2143.

(5] A. Aizpuru and M. Tamayo, 'Matrix convergence theorems in quantum logics', (preprint).

[6] P. Antosik and C. Swartz, Matrix methods in analysis, Lecture Notes in Mathematics 1113 (Springer-Verlag, Berlin, 1985).

[7] M.K. Bennett and D.J. Foulis, 'Effect algebras and unsharp quantum logics', Found. Phys. 24 (1994), 1331-1352.

[8] M.K. Bennett and D.J. Foulis, 'Interval and scale effect algebras', Adv. in Appl. Math. 19 (1997), 200-215.

[9] M.K. Birkhoff, Lattice theory 25, Colloquium New York (American Mathematical Society, 1948).

[10] M.K. Birkhoff and J. Von Neumann, 'The logic of quantum mechanics', Ann. Math. 37 (1936), 823-843. 
[11] S. Gudder, Quantum probability (Academic Press, London, New York, 1988).

[12] F.G. Mazario, 'Convergence theorems for topological group valued measures on effect algebras', Bull. Austral. Math. Soc. 64 (2001), 213-231.

[13] Z. Riecanova, 'Subalgebras, intervals and Central elements of generalized effect algebras', Internat. J. Theoret. Phys. 38 (1994), 3204-3220.

[14] J. Wu, S. Lu and D. Kim, 'Antosik-Mikusinski Matrix convergence theorem in quantum logics', Internat. J. Theoret. Phys. 42 (2003), 1905-1911.

[15] J. Wu, S. Lu and M. Chu, 'Quantum-logics-valued measure convergence theorem', Internat. J. Theoret. Phys 42 (2003), 2603-2608.

[16] J. Wu and Z. Ma, 'The Brooks-Jewett theorem on effect algebras with the sequential completeness property', Czechoslovak J. Phys. 53 (2003), 379-383.

Departamento de Matemáticas

Universidad de Cádiz

Apdo. 40

11510-Puerto Real (Cádiz)

Spain

e-mail: antonio.aizpuru@uca.es

marina.nicasio@uca.es

monserrat.tamayorivera@alum.uca.es 